\title{
Innovative Design of a Vertical and Transverse Elevator in Double Shafts, Based on TRIZ Theory
}

\author{
Jiwen Chen $1,2,{ }^{*}$ - Xin Li ${ }^{1,2}$ - Hongjuan Yang ${ }^{3}$ - Chen Wang ${ }^{1,2}$ \\ ${ }^{1}$ Shandong Jianzhu University, Co-Innovation Center for Green Building of Shandong Province, China \\ 2Shandong Jianzhu University, School of Mechanical and Electronic Engineering, China \\ 3Shandong Jianzhu University, School of Information and Electrical Engineering, China
}

With the rapid growth of urban populations, the scale of modern buildings is expanding, and the span of buildings is increasing. Traditional elevators can only solve the problem of vertical transportation. Passengers still need to spend time walking to their destination after leaving the elevator. Based on the theory of inventive problem solving (TRIZ), this paper uses functional models, causal analysis, conflict analysis, the Su-Field model, and other tools to innovate on the basis of traditional elevators. A vertical and transverse elevator design proposal for double shafts structures and corresponding control strategies is proposed. Based on C\#, simulations of the effect of the vertical and transverse elevator on the passenger mobility efficiency are conducted in an 8-story building at different transverse distances and densities. The simulation results show that the vertical and transverse elevator can effectively improve the mobility efficiency of passengers in the horizontal direction in the studied environment, and the mobility efficiency of all passengers also significantly improves. As the transverse distance increases, the mobility efficiency of all passengers using the proposed elevator also increases.

Keywords: TRIZ, Su-Field model, transverse elevator, Poisson distribution, elevator passengers' flow model

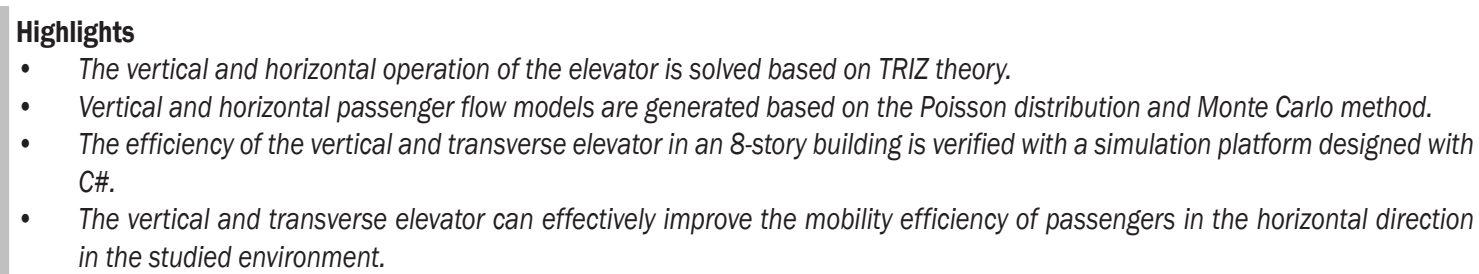

- $\quad$ The vertical and horizontal operation of the elevator is solved based on TRIZ theory.

- Vertical and horizontal passenger flow models are generated based on the Poisson distribution and Monte Carlo method.

- The efficiency of the vertical and transverse elevator in an 8-story building is verified with a simulation platform designed with C\#.

- $\quad$ The vertical and transverse elevator can effectively improve the mobility efficiency of passengers in the horizontal direction in the studied environment.

\section{INTRODUCTION}

Product innovation refers to the process of creating a new product or redesigning the function of a product [1]. To help engineers quickly generate innovative ideas to complete complex designs, many innovative methods have emerged. Commonly used innovative methods fall into two categories: intuitive methods and logical methods. Intuitive methods do not involve pre-analysis but produce innovative ideas by stimulating a dormant human thought process [2]. Because intuition is usually divergent, many detours generally occur. The logical method is based on the principles of science and engineering, and a large number of existing solutions must be analysed to systematically solve a problem. The theory of inventive problem solving (TRIZ) was proposed by Ahshuler, who analysed more than 2 million highlevel patents worldwide and proposed the involving invention, creation and technological innovation based on the principles of multiple disciplines [3]. Because TRIZ theory is highly abstract and can be repeatedly applied in different fields to solve different problems, it is now widely used as a scientific theory.
Elevators, as vertical transport tools in buildings, can solve the problem of vertical passenger transport in high-rise buildings and have been increasingly widely used [4]. Elevators are indispensable in modern highrise buildings and serve users with high efficiency and quality. With the rapid development of the economy and the rapid growth of the urban population, the scale of modern buildings is expanding, and the span of buildings is increasing [5]. Traditional elevators can only solve the problem of vertical transportation. Passengers still need to spend time walking to their destination after leaving the elevator. Because an elevator cannot move horizontally, it restricts the connections between different elevators with different shafts.

In 1996, the Otis Company first proposed the OdysseyTM elevator system, which uses transmission and traction drives in the vertical direction and a linear motor drive in the horizontal direction to achieve lateral elevator movement [6]. This system is mainly used to solve the transfer problems of elevators in different sections of high-rise buildings and can be applied to improve the mobility efficiency of passengers in large-span buildings. In [7], a rope- 
free elevator system based on planar positioners was proposed as an innovative solution for vertical and horizontal elevator movement based on the use of coordinate planar positioners as the drive source. In [8], the concept of a three-dimensional elevator system was introduced in a super-large building. The proposed virtual three-dimensional elevator system assumed that an elevator car can run in three directions (but obviously not at the same time). In [9], the Thyssen-Krupp Company's multi-elevator system was introduced and released in 2014. The cyclic multicar elevator system driven by a linear motor allows the vertical and horizontal operation of an elevator by installing steering devices at the nodes.

Although [6] to [9] effectively developed elevators that can achieve horizontal movement, due to technical or cost problems, these have not been effectively promoted. Thus, in this letter, we use the innovative principle introduced with the TRIZ theory to design vertical and transverse elevators in double shafts with low cost and broad applicability. To improve the passenger transport efficiency between distant shafts, a control strategy for an elevator with this structure is proposed and verified.

\section{TRIZ METHODS}

TRIZ provides a multifaceted approach to problemsolving and invention by treating the problem as a system and then trying to solve the related contradictions [10]. TRIZ includes a set of tools and methods for analysing and solving problems. The analysis tools are mainly used for the establishment, analysis and transformation of problem models, including causal analysis, Su-Field model analysis, conflict analysis, functional models as well as the algorithm for inventive problem solving (ARIZ) [11]. The problem-solving tools include inventive principles and scientific effect libraries [12]. These tools are developed by analysing a large number of highquality patents and learning from existing innovative experiences [13]. When applying TRIZ theory to solve practical engineering problems, first, it is necessary to effectively describe the problems, transform specific engineering problems into general TRIZ problems, and establish a TRIZ problem model [14]. Then, a suitable TRIZ tool is used to solve the problem model and obtain a general solution to the general problem. Finally, the specific solutions of engineering problems are obtained by mapping and comparison, and the solutions are verified and evaluated. When solving an engineering problem, we may use a tool or even multiple TRIZ tools.
Functional models can simplify the structure of technical systems and help engineers understand the interactions among components in technical systems in detail so that a few changes to the system can solve the system problems [15]. By analysing the functional models, we can specify the system function problems and determine the root causes of the problems through causal analysis. Using Su-Field model analysis, the nature of a problem can be determined by the type of problem described and then, referring to the transformation rules and 76 standard solutions of the Su-Field model, design ideas can be provided for designers [16].

In solving engineering problems, the most effective solution is to solve the contradictions in technical problems [17]. The understanding of contradictions and solutions to contradictions are very important concepts in TRIZ theory. In TRIZ, contradictions are divided into technical contradictions and physical contradictions. When improving one characteristic or parameter in a technical system, the contradiction that causes the deterioration of another characteristic or parameter in the system is called a technical contradiction [18]. A physical contradiction is a contradiction of the system itself. Thus, subsystems often have opposite requirements, and systems usually have only one contradiction parameter [19]. A physical contradiction often exists within a technical contradiction. A contradiction matrix is an important tool for solving technical contradiction problems. This matrix organically links 39 general engineering parameters with 40 inventive principles of TRIZ and establishes the corresponding relations. When dealing with technical contradictions, the problem is described

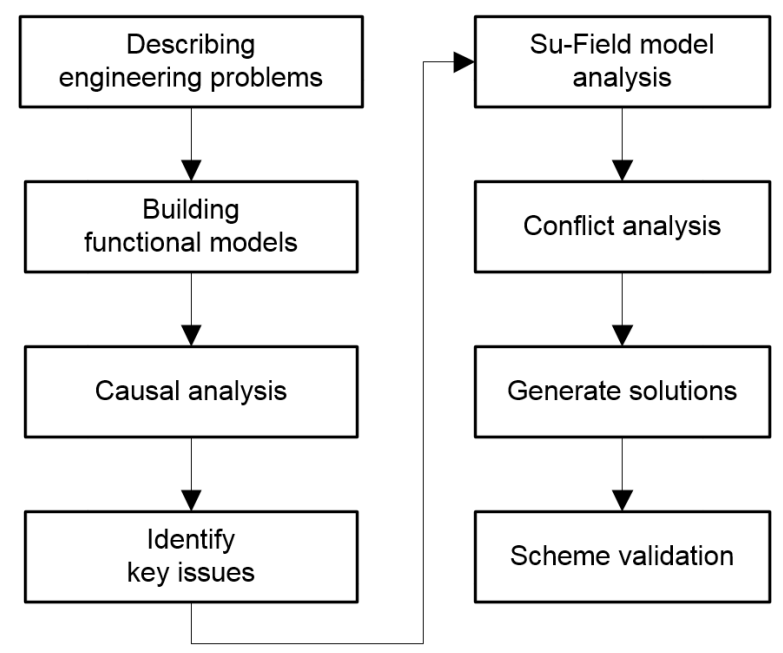

Fig. 1. Design process based on TRIZ 
as a conflict between any two of the 39 parameters [20]. The problem is solved by finding the inventive principle in the contradiction matrix. When dealing with physical contradictions, the separation principles can be used to solve problems.

The flow chart of solving the elevator horizontal movement problem is shown in Fig. 1. The key problems that must be solved are identified by functional models and causal analysis. The analysis and transformation problems are solved using the SuField model. Contradiction analysis is used to solve the problem and generate the solution. Then, the feasibility of the solution is verified by simulation experiments.

\section{DESIGN PROCESS}

\subsection{Functional Models}

Functional models describe the functions of technical and hyper-technical system components, as well as useful functions, performance levels, and cost levels [21]. In functional models, functions can be divided into useful functions and harmful functions. Useful functions include basic functions, auxiliary functions, and additional functions [22]. The performance level of a function is normal, excessive, insufficient or harmful. The graphical symbols are shown in Fig. 2.

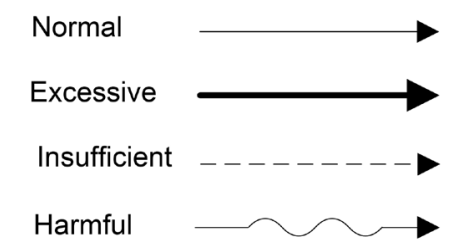

Fig. 2. Symbols of the performance levels of functions

Elevators are vertical transportation devices installed in building shafts that are mainly composed of a traction system, a guiding system, a door system, a weight balance system, and a safety protection system. The elevator core components, including the traction system, guidance system, and weight balance system, are selected for analysis. Then, the functions of the above components are summarized, and the function registration and performance level features are determined. The elevator system function model can then be established, as shown in Fig. 3.

By analysing the functional models, we find that there are two reasons that an elevator cannot move horizontally: 1. the elevator traction system cannot drive the car horizontally, and 2. the guiding device has a specific orientation to the car. To further explore the main causes of the problem, a causal analysis was performed for the above problem.

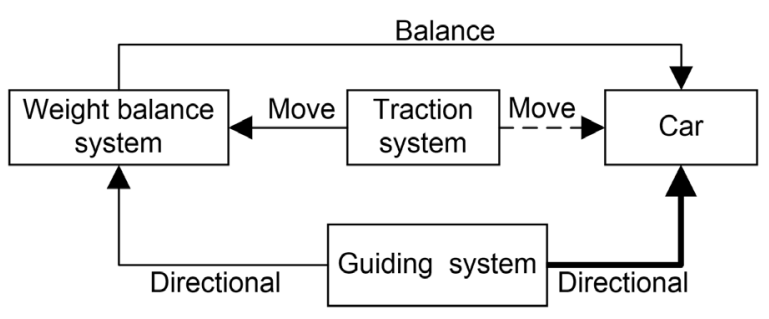

Fig. 3. Function model of an elevator

\subsection{Causal Analysis}

Causal analysis is a method of studying the relationship between development-based results and the causes of these results [23]. Starting from the problems that exist in a system, the causes of these phenomena are sequentially analysed, and the factors affecting the causality are analysed [24]. This paper chooses causal chain analysis starting from the problems to be solved, lists the direct causes of the problems, and uses these reasons as the results. Then, we search for the causes of these results and repeat the steps above until the root causes are found. Each cause and result are connected with arrows, and the arrow direction points from the cause to the result. When there are multiple causes of the problem, they are connected by "and" (multiple causes lead to results only if they exist at the same time) or "or" (multiple causes lead to results if only one exists). The causal chain model of a car that cannot move horizontally is shown in Fig. 4.

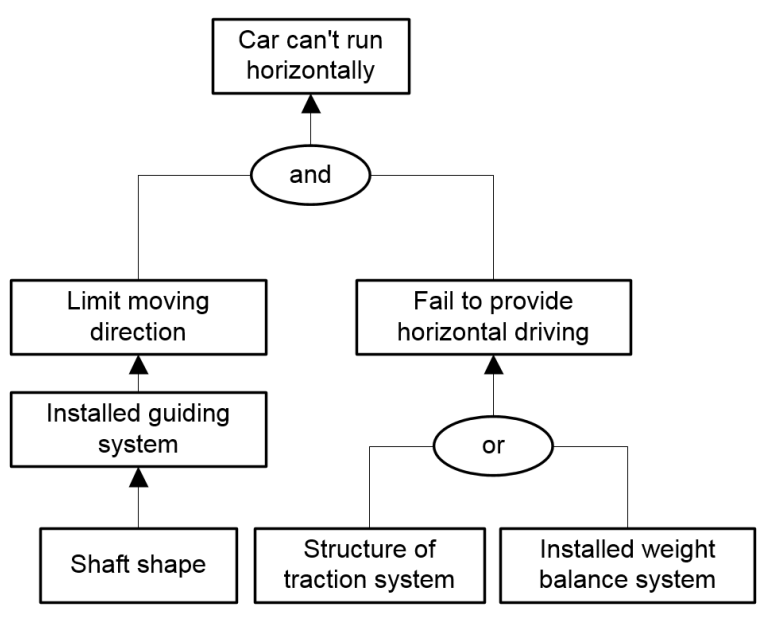

Fig. 4. Causal chain model of the problem

The causal chain model shows that the main reasons that the car cannot move horizontally are as 
follows: 1 the shaft shape is columnar and there is no space for horizontal movement, and 2. the structure of the traction system or the installed weight balance system does not allow the traction system to provide horizontal movement. After using causal analysis to find the root cause of the problem, the Su-Field model is used to model the problem, and the practical engineering problem is transformed into a general TRIZ problem.

\subsection{Su-Field Model Analysis}

The Su-Field model is the smallest complete system composed of two substances (S) and one field (F) [25]. The function of the system is expressed by the Su-Field triangle, which is composed of these three elements and clearly reflects the problems that exist in the system [26]. Typically, S1 acts as a recipient, S2 is the originator, and $\mathrm{F}$ is the interaction term. In the Su-Field model, the ideal state is F acting on S1 and changing S1 through S2 [27]. According to the theory of TRIZ, the Su-Field model of the minimal system of elevator car movement is constructed, and the relevant elements of the Su-Field model are determined as follows: S1 is the car; S2 is the traction system; and $\mathrm{F} 1$ is the mechanical force driving car movement, as shown in Fig. 5.

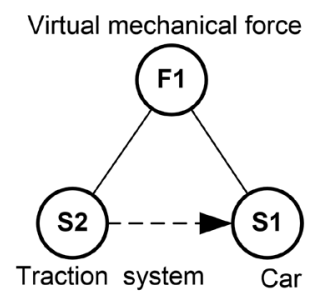

Fig. 5. Su-Field model of the minimal system of elevator car movement

According to TRIZ theory, the Su-Field model is analysed. The system is a complete Su-Field model with three complete elements. However, the car can only move in the vertical direction for transportation and not in the horizontal direction. A general solution of the Su-Field model was found: (1) replace the original field F1 with another field F2; (2) add another field F2 to enhance the practical effect; and (3) increase S3 and add another field F2 to enhance the effect [19]. Due to the lack of horizontal drive in the car system, solution (2) is selected to increase the horizontal driving force F2 on the car to strengthen the usefulness of the system, as shown in Fig. 6. However, due to the directional effect of the guiding system, the car is subjected to an excessive vertically guided mechanical force, and the vehicle still does not move horizontally. The Su-Field model in question is shown in Fig. 7.

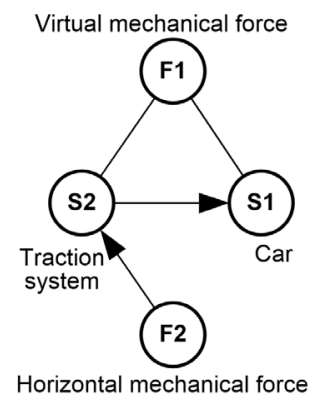

Fig. 6. Su-Field model after adding $F 2$

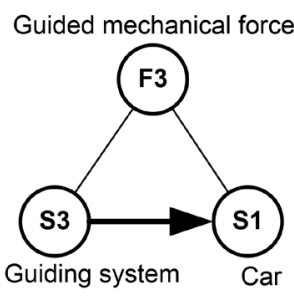

Fig. 7. Su-Field model of the guiding system
According to TRIZ theory, the Su-Field model is analysed, and the system is a complete Su-Field model with three elements, but the guiding device produces limits the orientation of the car, which yields a harmful complete model. The general solution of the look-up Su-Field model is as follows. (1) Add another substance S3 to prevent harmful effects. S3 can be an existing substance, variations of S1 and $\mathrm{S} 2$, or a substance obtained by decomposing the environment. (2) Add another field, F2, to balance the field that produces harmful effects [19].

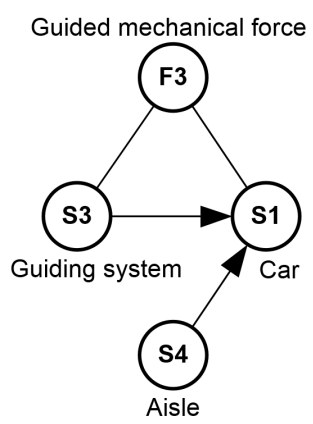

Fig. 8. Su-Field model after adding S4

According to the results of causal analysis, the orientation limitation of the car and the guiding system is caused by the shape of the shaft. Therefore, according to solution (1), aisle S4 is added to change the shape of the shaft, allow the car to move horizontally and overcome the excessive vertical guiding mechanical force of the guiding system, as shown in Fig. 8. When the car runs vertically in the shaft, it runs horizontally in the aisle to avoid influencing the car when it moves. The complete Su-Field model after eliminating insufficient and excessive performance factors is shown in Fig. 9. 


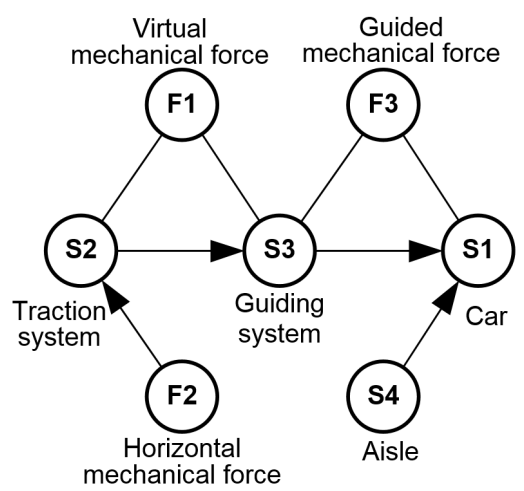

Fig. 9. Complete Su-Field model

\subsection{Conflict Analysis}

In the solution obtained from the analysis of the Su-Field model, the car should be able to complete both vertical and horizontal operations, and there is a problem associated with the car running direction that must be solved. Elevator operation requires both vertical and horizontal car operations, which are physical contradictions. Using separation principles is an important way to solve physical contradiction problems. TRIZ summarizes the separation principle into four categories: separation in space, separation in time, separation between the whole and its parts, and separation based on conditions [28]. Different separation principles correspond to different inventive principles.

After analysing the problem, the space separation principle is selected. Separation in space involves separating the two sides of a conflict in different spaces to reduce the difficulty of solving the problem. In the space separation principle [28], the available inventive principles are No. 1: segmentation, No. 2: taking out, No. 3: local quality, No. 4: asymmetry, No. 7: nested doll, No. 13: the other way round, No. 17: another dimension, No. 24: intermediary, No. 26: copying, and No. 30: flexible membranes / thin films. One or more of the given invention principles can be selected to solve the invention problem.

\subsection{Generate Solutions}

According to the analysis of physical contradictions, many inventive principles are obtained. Each inventive principle is a suggestion. By referring to these suggestions, the invention principle(s) most suitable for the current problem can be selected, the system can be changed, and the contradictions in the system can be eliminated [17]. According to the

analysis, the inventive principles most suitable for solving the elevator horizontal movement problem are selected, and the solutions are generated.

No. 1. Segmentation

Divide an object into independent parts.

The invention principle is analysed, and a design scheme is proposed to divide the car into two parts to run independently according to the direction of operation. The car is divided into a vertical car and a transverse car. The vertical car runs vertically in the elevator shaft. Only the passengers who need to move vertically are transported. The transverse car runs in the aisle and conveys passengers who need to move horizontally.

No. 24. Intermediary

Use an intermediary carrier article or intermediary process.

According to this invention principle, a design scheme is proposed to realize horizontal movement by adding a driving device at the bottom of the moving car with reference to rail-guided vehicle and metro systems. The wheel-rail system, which is widely applied in the field of rail transit, is used as the driving device and installed at the bottom of the transverse car. The track is laid on the running path of the transverse car. However, because passengers who require horizontal movement usually need to move vertically as well, the transverse car must also run vertically. Therefore, the driving system must be further designed for the transverse car.

\section{No. 2. Taking out}

Extract the only necessary part (or property) of an object.

According to this invention principle, a design scheme that allows the vertical driving of the transverse car using the existing traction system is proposed. In the current scheme, the vertical car runs vertically and is driven by the traction system, so the existing traction system is used to drive the transverse car to move vertically. When a transverse car needs to run vertically, it enters the shaft and performs vertical operations through the traction system. In this scheme, the connection between the transverse car and the existing traction system should be considered.

No. 17. Another dimension

Go from a single story or layer to a multi-storey or multilayer system.

No. 7. Nested doll

Place one object inside another. 
Based on the two inventive principles above, the design proposal of a double-decker car is proposed. The vertical car is divided into two layers. The upperdeck car is responsible for transporting the passengers who need to move vertically. The lower-deck car transports the passengers horizontally. A guide rail is installed at the bottom of the lower-deck car. When the transverse car needs to run vertically, it enters the lower-deck portion of the shaft from the passageway and runs vertically to the target layer driven by the driving traction system. The operation flow is shown in Fig. 10.

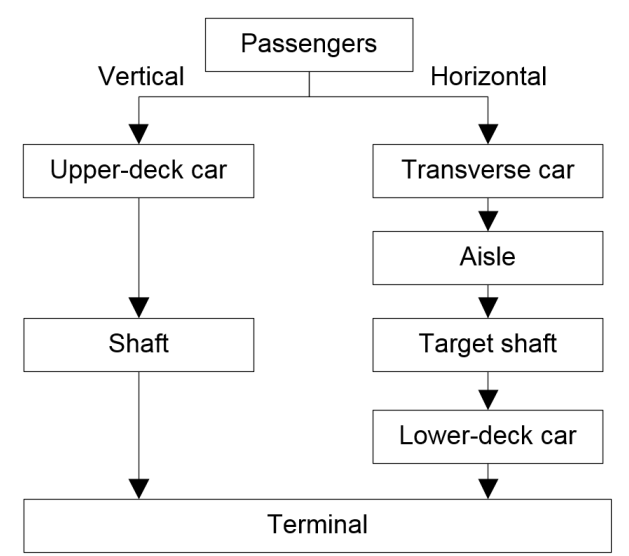

Fig. 10. Operation flow of the vertical and transverse elevator

However, in this scheme, there is often an angle at the junction between the aisle and the shaft where the car needs to be turned, as shown in Fig. 11. The size of the transverse car is relatively small, and the transverse car must run smoothly in the steering process to avoid passenger discomfort; therefore, an appropriate driving device for the transverse car must be designed.

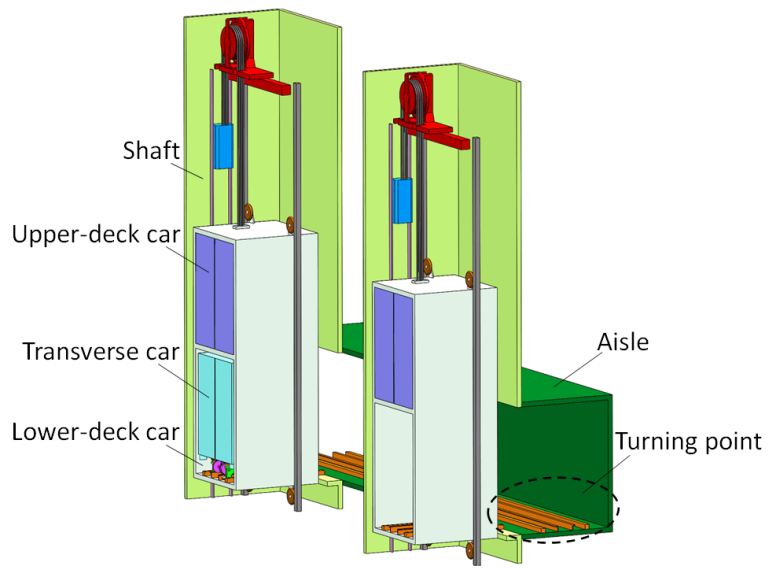

Fig. 11. Structures of buildings and elevators
No. 13: The other way round

Invert the action used to solve the problem.

The driving device of the transverse car must effectively perform driving and steering functions. The harmful factor is the steering function. When the car turns, the positions of the passengers in the car will also rotate, which will cause discomfort. According to this invention principle, a design scheme is proposed in which the main body of the car remains motionless while steering and only the driving device rotates. An active steering device and a fixing device are installed at the turning point of the passage, and a passively driven steering device is added to the transverse car. When the transverse car needs to change direction, the fixing device keeps the main body of the transverse car motionless, and the active steering device drives the passive steering device to rotate 90 degrees, changing the direction of motion of the driving device. The structures of the active steering device and fixing device are shown in Fig. 12. The structure of the transverse car is shown in Fig. 13. To ensure the safety of the transverse car, an anti-roll device and brake device are installed at the driving device. The structure of the driving device is shown in Fig. 14.

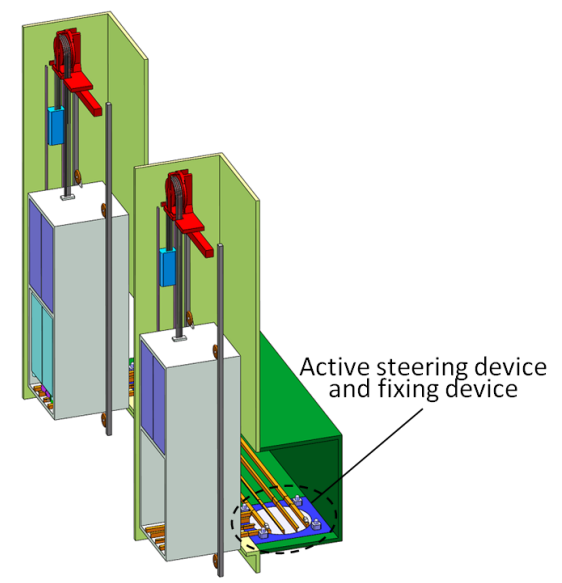

Fig. 12. The structure of the active steering device and fixing device

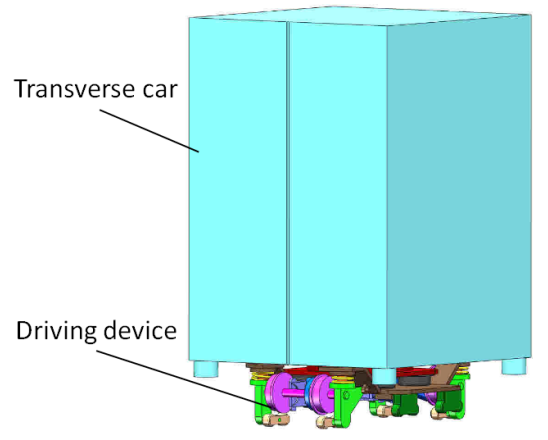

Fig. 13. The structure of the transverse car 


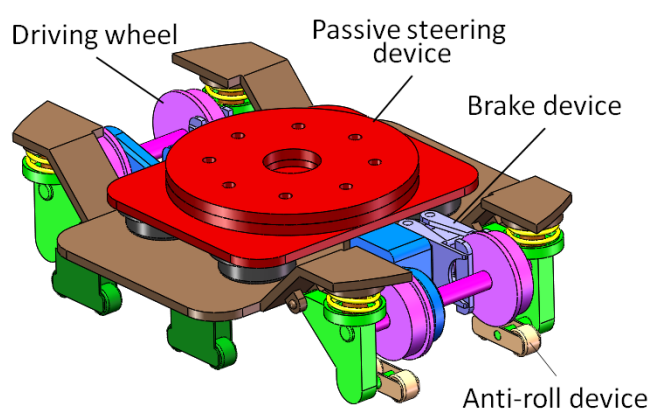

Fig. 14. The structure of the driving device

When the transverse car needs to move horizontally to the target shaft, it leaves the lowerdeck car and enters the turning point of the current shaft. Then, the transverse car moves horizontally in the aisle to the turning point of the target shaft path to change direction. The number of the aisle is according to the type of buildings and the number of passengers. Finally, the transverse car enters the lower-deck car of the target shaft. The transverse car is then driven vertically by the traction system, as shown in Fig. 15.

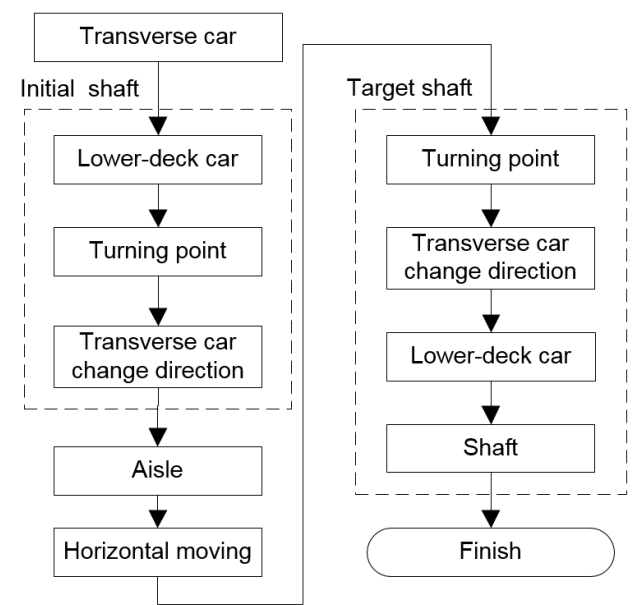

Fig. 15. Operation flow of vertical and transverse elevator

To further verify the design scheme obtained through TRIZ, a vertical and transverse elevator model is constructed, as shown in Fig. 16. The elevator model has two aisles, four floors, one transverse car and two shafts. The elevator control system is based on an Advanced RISC Machine (ARM). The stepping motor is used as the traction motor, and the transverse car receives and sends instructions by wireless communication. A digital steering gear is used to drive the active steering device, and an electromagnetic brake is installed in the passive steering device to ensure the accuracy of steering.

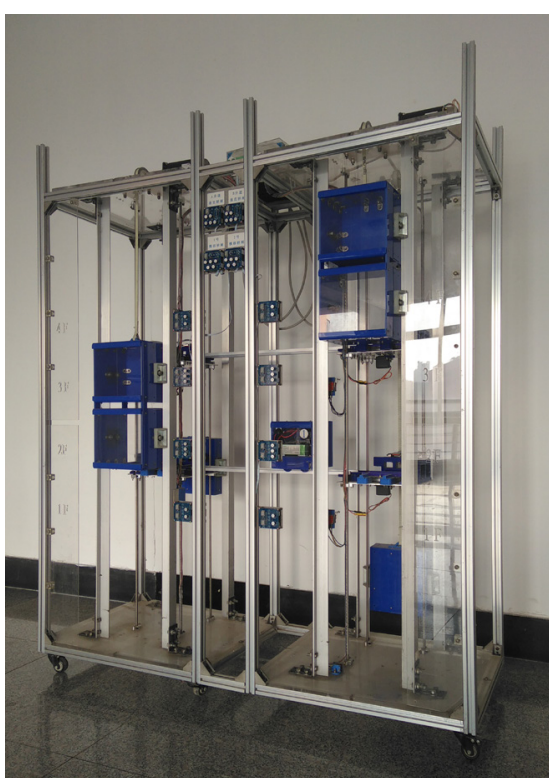

Fig. 16. Vertical and transverse elevator model

\section{SIMULATION VERIFICATION}

To verify the operational efficiency of the vertical and transverse lift elevator, the simulation of an 8-floor building is performed. Two shafts and two transverse cars are placed in the building, as shown in Fig. 17. The control strategy of the elevator is applied in this environment. The operational processes for the elevator are simulated with $\mathrm{C \#}$ by employing an object-oriented design and multithreading method.

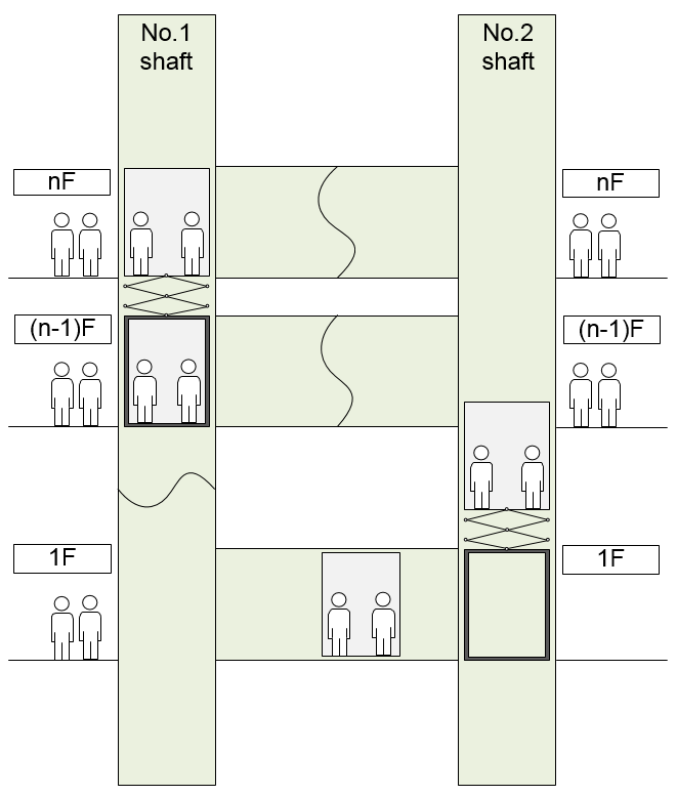

Fig. 17. Structures of buildings and elevators 


\subsection{Control Strategy}

The control strategy of vertical and transverse elevators should satisfy the real-time dispatching schemes of upper-deck and transverse cars, coordinate the operation of each car to avoid conflicts, and plan the optimal dispatching scheme for elevators [29]. To reduce the complexity of the control strategy, the transverse car is abstracted as a passenger using an elevator that can issue a landing call and a car call. The vertical cars in the two shafts operate independently and respond dynamically to the instructions of passengers and the transverse cars. It is necessary to control the horizontal movement of the transverse car to make it run smoothly and orderly in the aisle. Therefore, the control strategy of the vertical and transverse elevators is proposed. A horizontal call button is added to the call ladder panel. The horizontal call ladder does not distinguish between upward and downward directions. When the call signal for the transverse car is activated, the transverse car moves to the waiting area of the target shaft.

The variable $y$ is used to record the shaft number $(y=1,2)$. When the transverse car enters the lowerdeck car, variable $i$ is changed. The variable $k_{y}$ is used to record the upper-deck car on a given floor. Because the upper-deck car and the lower-deck car can answer the call at the same time, and the upper-car is one floor apart from the lower-car, the vertical control of the vertical and transverse elevator is determined relative to the upper-deck car on the floor. Sets are used to store transverse car and passenger instructions, as shown in Table 1. Because the control strategies of the cars in the No. 1 and No. 2 shafts are the same, only the control strategy in the No. 1 shaft $(y=1)$ is shown in Fig. 18.
Table 1. Detailed simulation parameters

\begin{tabular}{ll}
\hline Set & Function \\
\hline$V u_{y}$ & $\begin{array}{l}\text { Records upward passenger landing calls for vertical } \\
\text { transport }\end{array}$ \\
\hline$V d_{y}$ & $\begin{array}{l}\text { Records downward passenger landing calls for vertical } \\
\text { transport }\end{array}$ \\
\hline$V n_{y}$ & Records upper-deck car calls \\
\hline$H h_{y}$ & Records transverse car calls for horizontal transport \\
\hline$H v_{y}$ & Records transverse car calls for vertical transport \\
\hline$U_{y}$ & Records transverse car calls for lower-deck car \\
\hline$h_{i}$ & Records the floor where the transverse car is located \\
\hline
\end{tabular}

S1: The elevator operates vertically. When the upper-deck car satisfies the upward movement constraint and is located at the highest floor of the current call path, the direction of operation changes to the downward direction, as shown in Eq. (1):

$$
k_{1} \in \max \left(V u_{1} \cup U_{1} \cup H h_{1} \cup V n_{1}\right) .
$$

When the lower-deck car moves downward to the lowest floor of the current call instruction, the direction of operation changes to the upward direction, as shown in Eq. (2):

$$
k_{1} \in \min \left(V d_{1} \cup U_{1} \cup H h_{1} \cup V n_{1}\right) .
$$

S2: The elevator stops answering vertical calls when Eq. (3) is satisfied:

$$
k_{1} \in V u_{1} \cup V d_{1} \cup U_{1} \cup H h_{1} \cup V n_{1} .
$$

S3: Open and close the door of the upper-deck car. Load or unload the passengers who move vertically and update the instructions $V n_{1}$ for the upper-deck car.

S4: The transverse car answers calls from the ladder in the waiting area. When the transverse car arrives at the target shaft, it sends out a call and waits

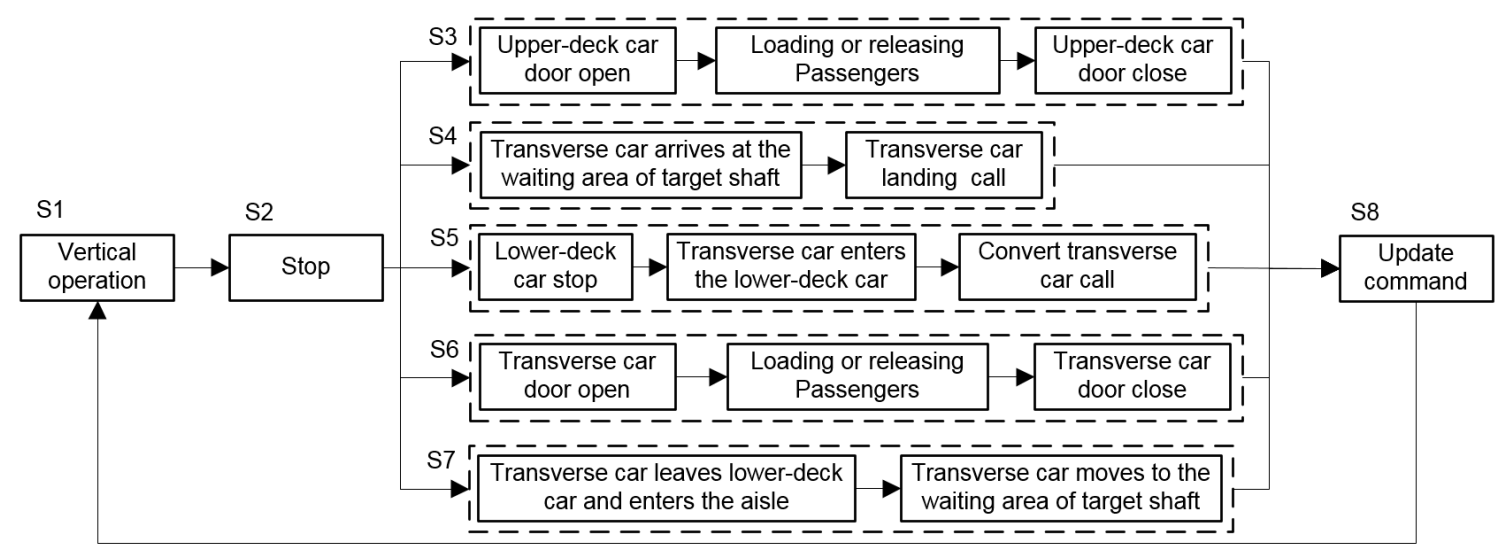

Fig. 18. Control strategy of vertical and transverse elevator 
for the response of the lower-deck car, as shown in Eq. (4):

$$
U_{1}=\left\{h_{2}\right\} .
$$

S5: The transverse car enters the lower-deck car of the target shaft. When the lower-deck car reaches the target floor, the transverse car enters the lowerdeck car and updates the number $i$ of the transverse car and converts the horizontal call of the transverse car to a vertical call of the transverse car in the current shaft, as shown in Eq. (5):

$$
H v_{1}=H h_{1} \text {. }
$$

The horizontal call of the transverse car is cleared, as shown in Eq. (6):

$$
H h_{1}=\varnothing \text {. }
$$

S6: Transverse car open and close the door. Load or release passengers who are running horizontally and update the transverse car call horizontal $H h_{1}$ and vertical $H v_{1}$.

S7: Transverse car into the aisle and moving horizontally. When there are no passengers to be released in the transverse car, as shown in Eq. (7):

$$
H v_{1}=\varnothing \text {, }
$$

and the transverse car is on the lowest floor or highest floor, as shown in Eq. (1) or Eq. (2), the transverse car enters the aisle of the current floor and moves to the waiting area of the target shaft. If the current floor aisle is occupied, the transverse car moves up or down one floor, and choose a free aisle.

S8: Update all instructions.

In addition, the control system monitors the position of each car and the passengers in the car in real time. When the upper-deck car or transverse car is overloaded, the overloaded car only responds to release passengers, and the non-overloaded car runs normally.

\subsection{Passengers' Flow Model}

Passengers taking the elevator is a random process that is commonly modelled as a stochastic process. Passenger requests for elevator service time are stochastic. The passenger-starting floor and target floor are uncertain. A Poisson process is a more reasonable arrival model for dynamic service systems [30]. Assuming that the arrival intervals of passengers adjacent to the elevator are independently, negatively, and exponentially distributed, the arrival process of passengers within 5 minutes obeys the Poisson process, and the passenger arrival rate $\lambda$ is as follows [31]:

$$
\begin{gathered}
\rho=\frac{(\lambda T)^{n} e^{-\lambda T}}{n !}, \quad(n=1,2,3 \ldots) . \\
\lambda=\frac{C E \times Q}{300},
\end{gathered}
$$

where $\rho$ is the probability of $n$ passengers requesting elevator service within period $T ; \lambda$ passenger arrival rate, i.e., the average number of passengers using the elevator per unit time; $C E_{1}$ number of passengers using an elevator within 5 minutes; and $Q$ total number of elevators in use.

Eq. (8) can be used to obtain the time between when passengers request and receive elevator service:

$$
\left\{\begin{array}{l}
t_{0}=A \\
t_{i}=t_{i-1}-\frac{\ln (r)}{\lambda},(i=1,2,3 \ldots)
\end{array},\right.
$$

where $t_{0}$ is the start time of the passenger service request, that is, the start time of the system simulation; $t_{i}$ first passenger service request for the elevator; and $r$ random number generated in the interval of $(0,1)$.

The Monte Carlo method is used to generate the starting and target floors of passengers in different traffic modes according to the proportions of up-peak, down-peak, and inter-floor passengers. The percentage of horizontal passengers to the total number of passengers is recorded as the transverse density. The numbers of horizontal and vertical passengers are determined by the Monte Carlo method.

\subsection{Simulation Platform}

To study the elevator's effect of the vertical and traversing on the passenger mobility efficiency, a vertical and transverse elevator simulation platform was developed. The simulation platform was designed with $\mathrm{CH}$ and used a multithreading method to simulate the operation of vertical and transverse elevators. The simulation platform mainly includes an interactive interface, a passengers' flow simulation module, an elevator operation module and a data statistics module. The simulation interface is mainly used to configure the parameters of each module and display the running status of the elevator. The passengers' flow simulation module generates passengers' flows under different traffic modes according to the Poisson distribution and Monte Carlo method. In the same passengers' flow environment, the elevator operation module simulates the operation of the vertical and transverse elevator 


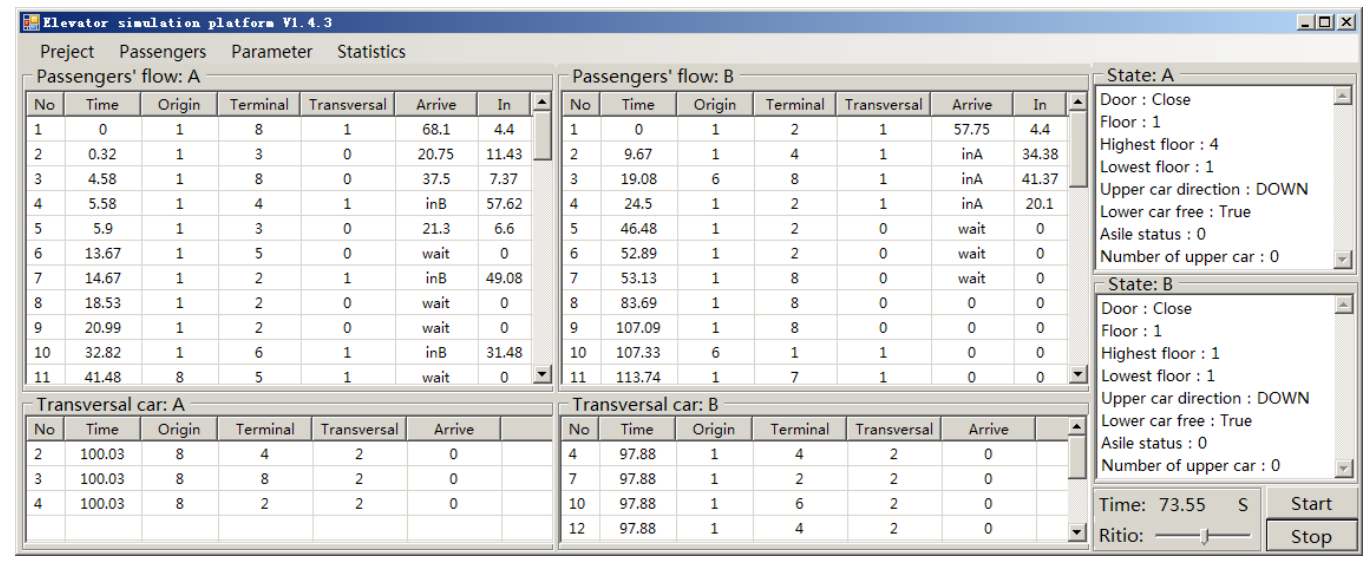

Fig. 19. Elevator simulation platform

and the ordinary elevator. The ordinary elevator is a single-deck car elevator without an installed aisle. The data statistics module is responsible for recording the operation parameters and times of elevator operations and calculating the average waiting time, average riding time, and passenger mobility efficiency. The elevator simulation platform is shown in Fig. 19.

\subsection{Simulation Case}

Vertical and transverse elevators are arranged in an eight-floor building. The system consists of two vertical cars and two transverse cars. In total, 640 passengers are distributed evenly among the eight floors, generating $300 \mathrm{~s}$ of passengers' flow data per simulation, and the elevator simulation parameters are shown in Table 2. The simulation is conducted in the up-peak, down-peak, and inter-floor traffic modes. The configuration of traffic modes is shown in Table

Table 2. Detailed simulation parameters

\begin{tabular}{lc}
\hline Parameters & Value \\
\hline Story height & $4 \mathrm{~m}$ \\
\hline Number of elevator users & 640 \\
\hline Aisle length & $200 \mathrm{~m}$ \\
\hline Passenger arrival rate & 0.128 \\
\hline Vertical speed of the elevator & $1.5 \mathrm{~m} / \mathrm{s}$ \\
\hline Horizontal speed of the elevator & $8 \mathrm{~m} / \mathrm{s}$ \\
\hline Acceleration & $1.5 \mathrm{~m} / \mathrm{s}^{2}$ \\
\hline Passenger walking speed & $1 \mathrm{~m} / \mathrm{s}$ \\
\hline Number of upper-deck car & 10 \\
\hline Number of transverse cars & 10 \\
\hline Opening/closing door time & $1 \mathrm{~s}$ \\
\hline Entry/exit time for each passenger & $0.5 \mathrm{~s}$ \\
\hline
\end{tabular}

3. By comparing the arrival times of multi-direction elevator passengers and ordinary elevator passengers based on the same parameters, the influence of the newly proposed elevator on the passenger mobility efficiency is obtained. The 'averagesimulation' code was executed five times for each group to ensure the accuracy of the experiment.

Table 3. Distribution of different traffic modes

\begin{tabular}{lccc}
\hline & Up-peak & Inter-floor & Down-peak \\
\hline Upward ratio [\%] & 80 & 10 & 10 \\
\hline Inter-floor ratio [\%] & 10 & 80 & 10 \\
\hline Downward ratio [\%] & 10 & 10 & 80 \\
\hline
\end{tabular}

The effect of the vertical and transverse elevator on the arrival efficiency of all passengers is shown in Fig. 20, and the effect on the arrival efficiency of horizontal-moving passengers is shown in Fig. 21. The data analysis indicated that the efficiency of horizontal-moving passengers was significantly improved by adding the transverse car, and the total efficiency of all passengers also significantly improved. The efficiency of the vertical and transverse elevator was high, especially in the up-peak and interfloor traffic modes.

Additional data analysis indicated that the run of the transverse car will have an impact on the vertical passengers. The average waiting times of vertical passengers for the ordinary elevator and vertical and transverse elevators are compared for different transverse densities, as shown in Fig. 22. In downpeak traffic mode, the vertical and transverse elevator has an obvious impact on the average waiting time of vertical passengers, and lesser effects are observed in the up-peak and inter-floor traffic modes. 


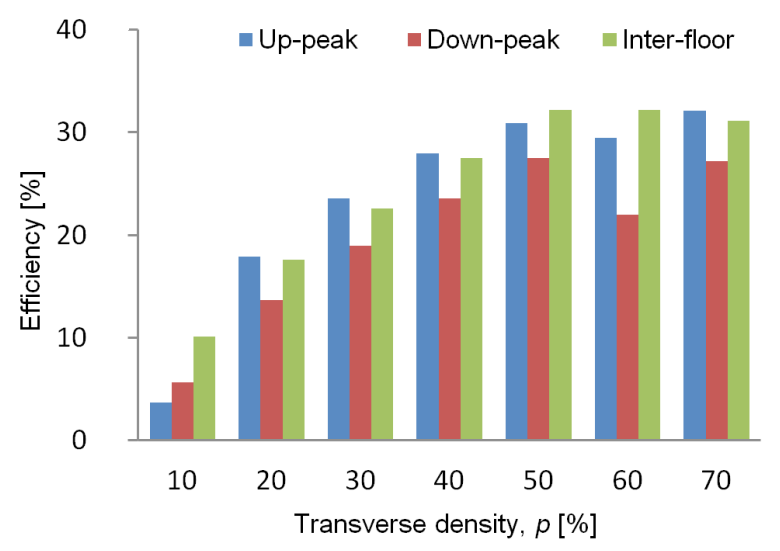

Fig. 20. The arrival efficiency of all passengers

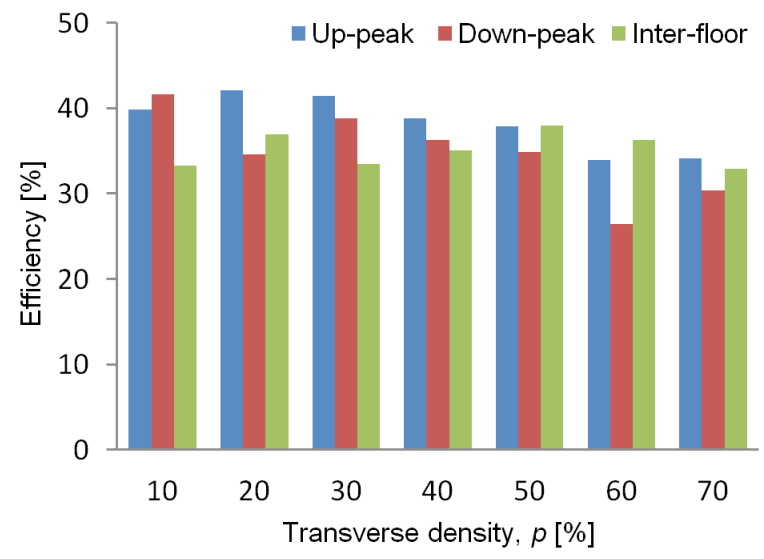

Fig. 21. The arrival efficiency of horizontal moving passengers

To study the influence of the transverse distance on the vertical and transverse elevator effectiveness, the transverse distance is set to 300 metres and 400 metres. In the simulation, other parameters remain unchanged. The transverse distances are 200 metres, 300 metres and 400 metres, and the effect of the vertical and transverse elevator on the arrival efficiency of all passengers is shown in Fig. 23.
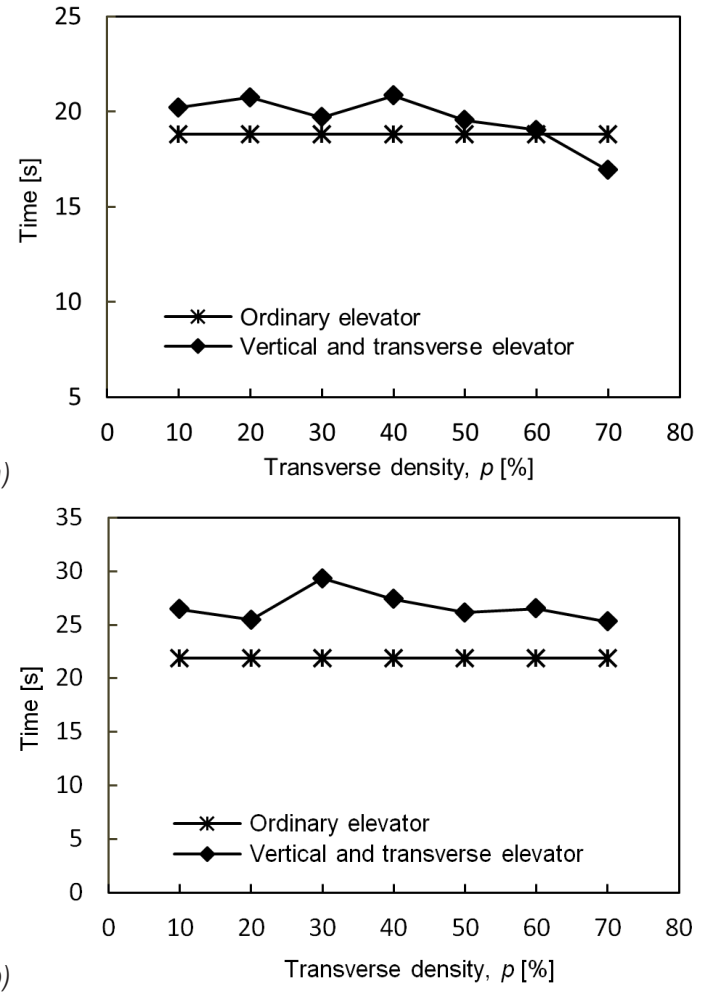

b)

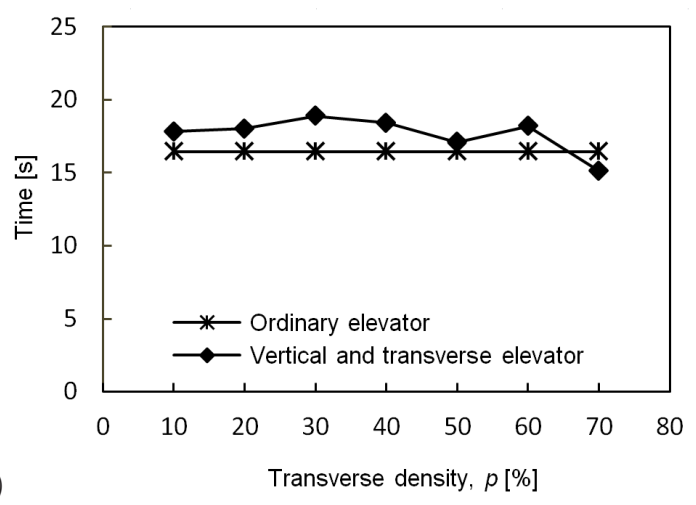

Fig. 22. Comparison of average waiting time for vertical passengers when the aisle is 200 meters; a) up-peak traffic mode, b) inter-floor traffic mode, and c) down-peak traffic mode

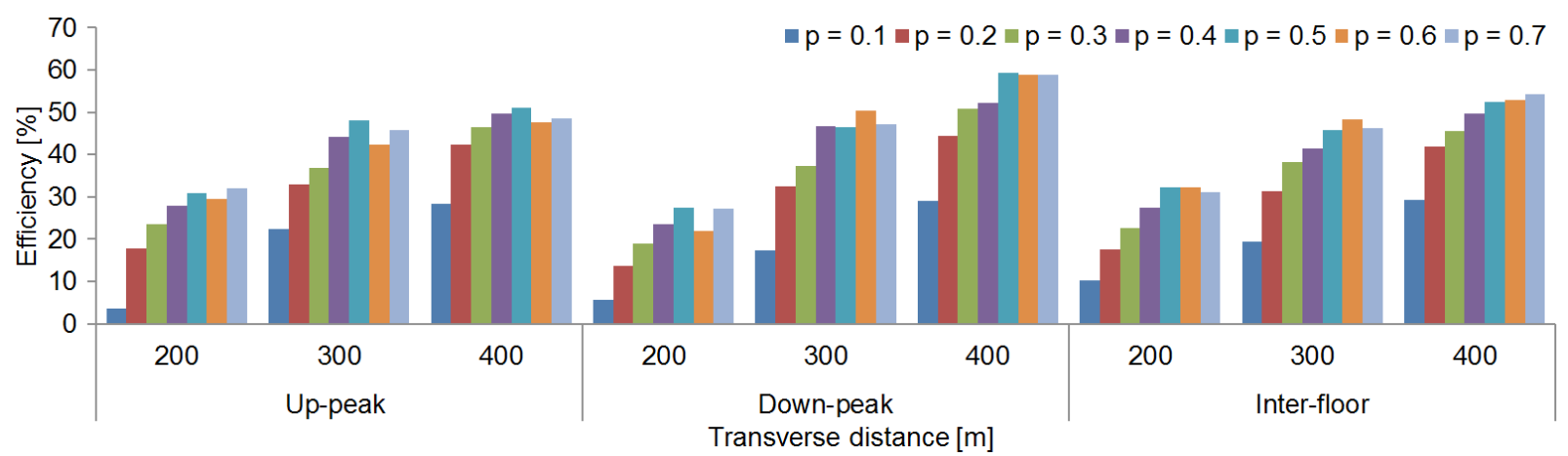

Fig. 23. The arrival efficiency of all passengers when the transverse distance is 200,300 and 400 meters 
As the transverse distance increases, the efficiency of the vertical and transverse elevator for all passengers increases. The average waiting times of vertical passengers for the ordinary elevator and vertical and transverse elevator are compared for a transverse distance of 400 metres, as shown in Fig. 24. A comparison of Figs. 22 and 24 shows that the average waiting time of vertical passengers does not significantly increase as the transverse distance of the vertical and transverse elevator increases.
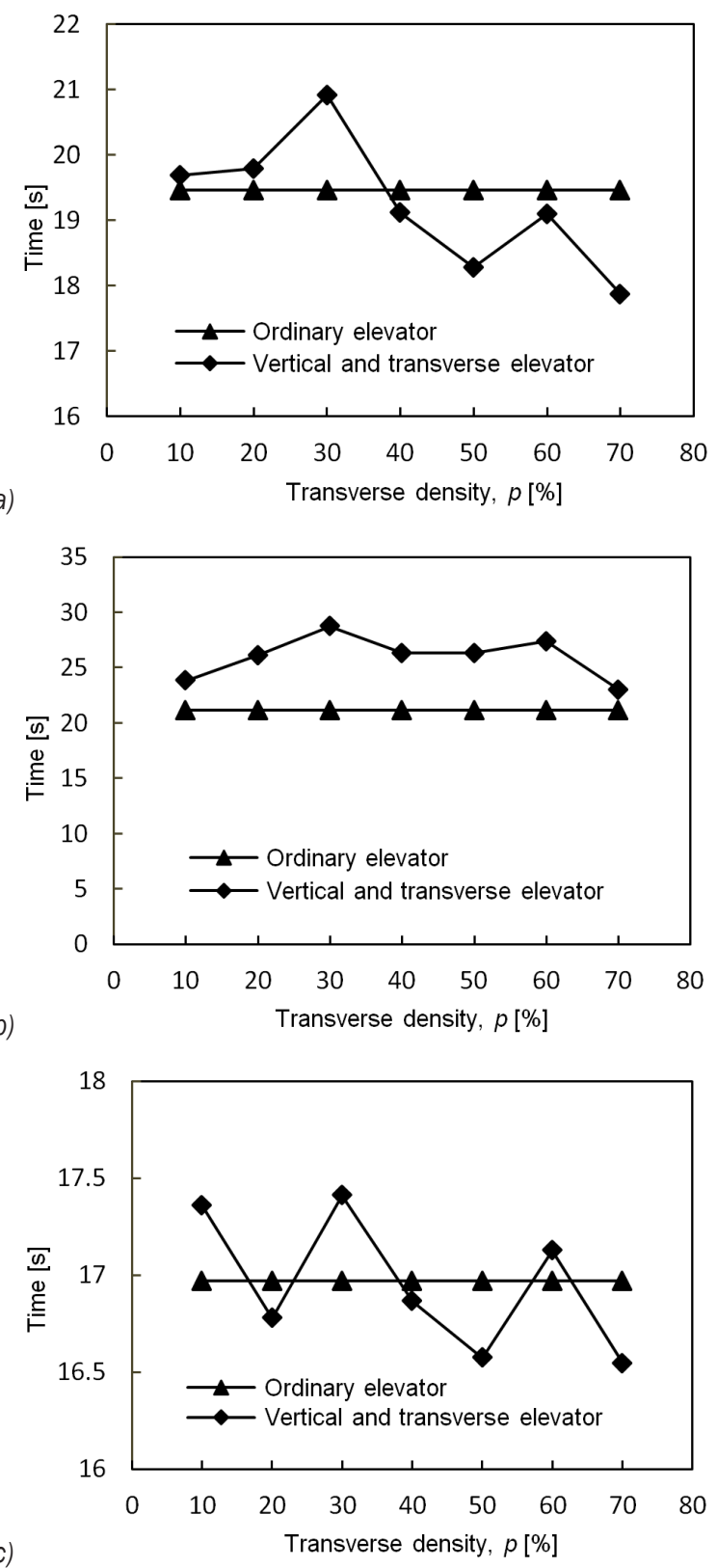

Fig. 24. Comparison of average waiting times for vertical passengers when the aisle is 400 meters; a) up-peak traffic mode, b) inter-floor traffic mode, and c) down-peak traffic mode

\section{DISCUSSION}

In the above cases, the efficiency of the vertical and transverse elevator for horizontal passengers is significantly improved, mainly because the passengers using the ordinary elevator must walk horizontally to their destination and the horizontal moving speed of the transverse car is much higher than that of the walkers. However, the operation of the transverse car also has an impact on the efficiency of the vertical passengers, especially in the down-peak traffic mode, as shown in Figs. 22b and 24b. Among the three traffic modes, passengers in the down-peak traffic mode are more dispersed on the initial and target floors. As a result, the number of transverse car stops required to answer the calls in the vertical direction is greater than that in other cases, so the average waiting time for vertical passengers has a notable impact on the overall system efficiency. However, compared with the improvement in the horizontal mobility efficiency of passengers, the impact on the vertical passengers is negligible.

In the cross-control strategy of the vertical and transverse elevator, when the transverse car responds to the passenger elevator signal, it does not distinguish between the movement directions of the passenger (i.e., horizontal vs vertical). However, the passenger in an ordinary elevator must wait when the elevator runs in the direction opposite their direction of travel. Therefore, under certain conditions, the average vertical passenger time of the vertical and transverse elevator may be less than that of the ordinary elevator. As shown in Fig. 24a, the average waiting time of vertical passengers using the vertical and transverse elevator is lower than that of the ordinary elevator when the transverse distance is 400 metres, and the transverse density $p$ is greater than 0.4 . Therefore, in the future, we can conduct in-depth research on this phenomenon and optimize the control strategy in down-peak traffic mode to reduce the average waiting time of vertical passengers and improve operational efficiency.

\section{CONCLUSIONS}

Based on TRIZ theory, this paper uses functional models, causal analysis, conflict analysis, the SuField model and other tools to innovate on the basis of traditional elevators. A vertical and transverse elevator design proposal for a double shafts structure is proposed. The control strategy is designed according to the vertical and transverse elevator structure design. A simulation platform for the elevator structure is 
developed with $\mathrm{C} \#$ to investigate the operational efficiency for different traffic modes and transverse distances. The simulation results show that the vertical and transverse elevator can effectively improve the mobility efficiency of passengers in the horizontal direction in the pre-set environment, and the mobility efficiency of all passengers also significantly improves.

\section{ACKNOWLEDGEMENTS}

The authors would like to thank the support of the National Natural Science Foundation of China (Grant No. 61303087), the Key R \& D project of Shandong Province (Grant No. 2016GGX103042) and Innovation Team Support Project of Co-Innovation Center for Green Building of Shandong Province in Shandong Jianzhu University (No. X18024Z). Jiwen Chen and Xin Li contributed equally to this work.

\section{REFERENCES}

[1] Chai, K.-H., Zhang, J., Tan, K.-C. (2005). A TRIZ-based method for new service design. Journal of Service Research, vol. 8, no. 1, p. 48-66, DOI:10.1177/1094670505276683.

[2] Lin, S.Y., Wu, C.T. (2016). Application of TRIZ inventive principles to innovate recycling machine. Advances in Mechanical Engineering, vol. 8, no. 5, p. 1-8, DOI:10.1177/1687814016647303.

[3] Altshuller, G.S. (1984). Creativity as Exact Science: The Theory of the Solution of Inventive Problems. Gordon \& Breach Science Publishers, London.

[4] Ikuta, M., Takahashi, K., Inaba, M. (2013). Strategy selection by reinforcement learning for multi-car elevator systems. IEEE International Conference on Systems, Man, and Cybernetics, p. 2478-2484, DOI:10.1109/SMC.2013.423.

[5] So, A., Al-Sharif, L., Hammoudeh, A. (2016). Concept design and derivation of the round trip time for a general two-dimensional elevator traffic system. Journal of Building Engineering, vol. 5, p. 165-177, D0l:10.1016/j. jobe.2015.12.006.

[6] Barker, F.H. (1997). A technical primer: the Otis odyssey system. Proceedings of International Conference on Council on Tall Buildings and Urban Habitat, p. 55-76.

[7] Trochimczuk, R., Huścio, T. (2017). Rope-free elevator system based on planar positioners for vertical and horizontal transport. Mechanika, vol. 23, no. 1, p. 120-125, DOI:10.5755/j01.mech.23.1.13868.

[8] So, A., Al-Sharif, L., Hammoudeh, A. (2018). Traffic analysis of a three-dimensional elevator system. Building Services Engineering Research and Technology, vol. 39, no. 1, p. 5-20, DOI:10.1177/0143624417710106.

[9] Appunn, R., Frantzheld, J., Jetter, M., Löser, F. (2018). MULTI® - rope-less elevator demonstrator at test tower Rottweil. Transportation Systems and Technology, vol. 4, no. 3, p. 8089, D0I:10.17816/transsyst20184380-89.
[10] Srinivasan, R., Kraslawski, A. (2006). Application of the TRIZ creativity enhancement approach to design of inherently safer chemical processes. Chemical Engineering \& Processing: Process Intensification, vol. 45, no. 6, p. 507-514, D0I:10.1016/j.cep.2005.11.009.

[11] Liu, W., Cao, G., Tan, R. (2016). Research on optimization of TRIZ application driven by design needs and targets. Procedia CIRP, vol. 39, p. 33-38, D0I:10.1016/j.procir.2016.01.162.

[12] Petković, D., Issa, M., Pavlović, N.D., Zentner, L. (2013). Application of the TRIZ creativity enhancement approach to the design of a passively adaptive compliant robotic gripper. Assembly Automation, vol. 33, no. 3, p. 231-239, DOI:10.1108/AA-10-2013-079.

[13] Gronauer, B., Naehler, H. (2016). TRIZ as an amplifier for corporate creativity and corporate innovation ability. Procedia CIRP, vol. 39, p. 185-190, D0l:10.1016/j.procir.2016.01.186.

[14] Wang, Y.-H., Lee, C.-H., Trappey, A.J.C. (2017). Service design blueprint approach incorporating TRIZ and service QFD for a meal ordering system: A case study. Computers \& Industrial Engineering, vol. 107, p. 388-400, D0l:10.1016/j. cie.2017.01.013.

[15] Efimov-Soini, N.K., Chechurin, L.S. (2016). Method of ranking in the function model. Procedia CIRP, vol. 39, p. 22-26, DOl:10.1016/j.procir.2016.01.160.

[16] Mi, J.S., Lee, J.-G., Park, J.-M., Lee, S. (2012). Triggering navigators for innovative system design: the case of lab-on-achip technology. Expert Systems with Applications, vol. 39, no. 16, p. 12451-12459, D0l:10.1016/j.eswa.2012.04.068.

[17] Cho, C.-H. , Chae, S.-W., Kim, K.-H. (2014). Search for a new design of deburring tools for intersecting holes with TRIZ. The International Journal of Advanced Manufacturing Technology, vol. 70, no. 9-12, p. 2221-2231, D0l:10.1007/s00170-0135459-y.

[18] Petković, D., Issa, M., Pavlović, N.D., Zentner, L. (2013). Application of the triz creativity enhancement approach to design of passively compliant robotic joint. International Journal of Advanced Manufacturing Technology, vol. 67, no. 1-4, p. 865-875, D0l:10.1007/s00170-012-4530-4.

[19] Savransky, S.D. (2002). Engineering of Creativity. CRC Press, New York.

[20] Hsieh, H.T., Chen, J.L. (2010). Using TRIZ methods in friction stir welding design. International Journal of Advanced Manufacturing Technology, vol. 46, no. 9-12, p. 1085-1102, DOI:10.1007/s00170-009-2172-y.

[21] Cao, G.Z., Guo, H.X., Liang, T., Tan, R.H. (2011). Function modeling, solving and simulation based on effect in TRIZ. Advanced Materials Research, vol. 418-420, p. 2190-2194, Dol:10.4028/www.scientific.net/AMR.418-420.2190.

[22] Busov, B., Mann, D., Jirman, P. (1999). Case studies in TRIZ: a novel heat exchanger (use of function analysis modelling to find and eliminate contradictions). The 3rd European Invention Machine User Group Conference, Munich.

[23] Aurisicchio, M., Bracewell, R., Armstrong, G. (2013). The function analysis diagram: intended benefits and coexistence with other functional models. Artificial Intelligence for Engineering Design, Analysis and Manufacturing, vol. 27, no. 3, p. 1-26, DOI:10.1017/S0890060413000255. 
[24] Liu, W., Cao, G., Tan, R. (2016). Research on optimization of TRIZ application driven by design needs and targets. Procedia CIRP, vol. 39, p. 33-38, D0l:10.1016/j.procir.2016.01.162.

[25] Cui, S.-h., Mei, J.-p., Zhang, L., Du, X. (2017). Based on the theory of TRIZ solving the problem of 18650 battery electrolyte filling. IOP Conference Series: Earth and Environmental Science, p. 1-5, Dol:10.1088/1755-1315/104/1/012018.

[26] Chou, J.R. (2014). An ideation method for generating new product ideas using TRIZ, concept mapping, and fuzzy linguistic evaluation techniques. Advanced Engineering Informatics, vol. 28, no. 4, p. 441-454, D0l:10.1016/j. aei.2014.06.006.

[27] Fan, D.L., Hu, J.W., Yu, H.L. (2011). Application of Su-Field analysis method to the innovation of decision-making.
Applied Mechanics and Materials, vol. 130-134, p. 135-138, DOI:10.4028/www.scientific.net/AMM.130-134.135.

[28] Hipple, J. (2012). TRIZ Separation Principles. In: The Ideal Result. Springer, New York.

[29] Tanaka, S., Hoshino, D., Watanabe, M. (2016). Group control of multi-car elevator systems without accurate information of floor stoppage time. Flexible Services and Manufacturing Journal, vol. 28, no. 3, p. 461-494, Dol:10.1007/s10696-0169238-6.

[30] So, A.T., Chan, W.L. (2009). Intelligent Building Systems. Johnson Controls Asia, Hong Kong.

[31] Pan, Z., Luo, F., Xu, Y. (2007). Elevator traffic flow model based on dynamic passenger distribution. IEEE International Conference on Control and Automation, p. 2386-2390, DOI:10.1109/ICCA.2007.4376789. 